\title{
When are two Markov chains similar?
}

\author{
Brian Fralix \\ Department of Mathematical Sciences \\ Clemson University \\ Clemson, SC, USA \\ bfralix@clemson.edu
}

August 24, 2015

\begin{abstract}
We present sufficient conditions for two Markov chains to be similar, thus confirming a conjecture of Pollett (J. Appl. Prob 38A (2001), 53-65). Our proof technique also shows one of the sufficient conditions given in Theorem 2 of Lenin et al. (J. Appl. Prob 37 (2000), 835-849) for two birth-death processes to be similar is unnecessary.
\end{abstract}

Keywords: similar Markov chain, transient behavior, transition function MSC: $60 \mathrm{~J} 27,60 \mathrm{~J} 35$.

\section{Introduction}

This note addresses the question of when two continuous-time Markov chains (CTMCs) are similar, as defined in Lenin et al. [3] and Pollett [4].

Suppose $X:=\{X(t) ; t \geq 0\}$ and $\bar{X}:=\{\bar{X}(t) ; t \geq 0\}$ are two CTMCs having state space $E$, and rate matrices $\mathbf{Q}:=[q(x, y)]_{x, y \in E}$ and $\overline{\mathbf{Q}}:=[\bar{q}(x, y)]_{x, y \in E}$, respectively. Further associated with $X$ is its collection of transition times $\left\{T_{n}\right\}_{n \geq 0}$, where $T_{0}:=0$, as well as its embedded discrete-time 
Markov chain (DTMC) $\left\{X_{n}\right\}_{n \geq 0}$, where $X_{n}:=X\left(T_{n}\right)$ for each integer $n \geq 0$. Similarly, we let $\left\{\bar{T}_{n}\right\}_{n \geq 0}$ and $\left\{\bar{X}_{n}\right\}_{n \geq 0}$ represent the transition times and the embedded DTMC associated with $\bar{X}$.

We assume $X$ and $\bar{X}$ share the same transition structure, meaning for any pair of states $x, y \in E$, $x \neq y, q(x, y)>0$ if and only if $\bar{q}(x, y)>0$. This assumption ensures both chains share the same communicating classes. Furthermore, we do not force either $X$ or $\bar{X}$ to be regular, meaning either chain could explode in a finite amount of time: to model such a phenomenon, we create an additional state $\Delta$ outside of $E$, we let $T:=\lim _{n \rightarrow \infty} T_{n}$ represent the time at which $X$ explodes, and we define

$$
X(t)= \begin{cases}\sum_{n=0}^{\infty} X_{n} \mathbf{1}\left(T_{n} \leq t<T_{n+1}\right), & t<T \\ \Delta, & t \geq T\end{cases}
$$

with $\bar{X}$ being constructed analogously, so that both processes are well-defined on $[0, \infty)$. Finally, we assume both $X$ and $\bar{X}$ are stable and conservative over $E$, meaning $\mathbf{Q}$ satisfies $q(x):=-q(x, x)=$ $\sum_{y \neq x} q(x, y)<\infty$ for each $x \in E$, and similarly for $\overline{\mathbf{Q}}$.

It is known - see e.g. Section 2.2 of Anderson [1] - that the transition functions $\left\{p_{x, y}\right\}_{x, y \in E}$ associated with $X$ are the minimal transition functions that solve the corresponding Kolmogorov forward and backward equations, where for each $t \geq 0, x, y \in E$,

$$
p_{x, y}(t):=\mathbb{P}_{x}(X(t)=y)
$$

with $\mathbb{P}_{x}$ representing a conditional probability, given $X(0)=x$. Throughout we will still use the notation $\mathbb{P}_{x}$ when conditioning on other Markov chains being in state $x$ at time zero, with $\mathbb{E}_{x}$ being the corresponding expectation: this usage of notation is fairly standard, and should not cause any additional confusion. For example, for each $x, y \in E$ we follow the convention that the transition function $\bar{p}_{x, y}$ associated with $\bar{X}$ evaluated at time $t, \bar{p}_{x, y}(t)$, can also be expressed as $\mathbb{P}_{x}(\bar{X}(t)=y)$.

Our goal is to find sufficient conditions for $X$ and $\bar{X}$ to be similar over a common communicating class $C$, where similarity is defined as follows.

Definition 1.1 We say $X$ and $\bar{X}$ are similar over a communicating class $C$ if there exists a collec- 
tion of positive numbers $\left\{c_{x, y}\right\}_{x, y \in C}$ satisfying, for each $x, y \in C$,

$$
\bar{p}_{x, y}(t)=c_{x, y} p_{x, y}(t), \quad t \geq 0 .
$$

The next theorem is the main result of this note: it will be proven in Section 3.

Theorem 1.1 Suppose that for some communicating class $C$ of both $X$ and $\bar{X}$, the following conditions are satisfied:

(a) for each $x \in C, q(x)=\bar{q}(x)$.

(b) for each $x \in C$, every feasible path $x=x_{0}, x_{1}, \ldots, x_{n-1}, x_{n}=x$ under $\boldsymbol{Q}$ (and also $\overline{\boldsymbol{Q}}$ ) starting and ending at $x$ satisfies

$$
\prod_{\ell=1}^{n} q\left(x_{\ell-1}, x_{\ell}\right)=\prod_{\ell=1}^{n} \bar{q}\left(x_{\ell-1}, x_{\ell}\right)
$$

Then $X$ and $\bar{X}$ are similar over $C$.

The next lemma provides a simple, yet important consequence of Conditions (a) and (b) and will be used in Section 3 to prove Theorem 1.1.

Lemma 1.1 Suppose Condition (b) of Theorem 1.1 holds, and let $x, y$ be two distinct states in $C$. Then for any two feasible paths $x=x_{0}, x_{1}, \ldots, x_{m-1}, x_{m}=y$ and $x=y_{0}, y_{1}, \ldots, y_{n-1}, y_{n}=y$ under $Q$ from $x$ to $y$, we have

$$
\prod_{\ell=1}^{m} \frac{\bar{q}\left(x_{\ell-1}, x_{\ell}\right)}{q\left(x_{\ell-1}, x_{\ell}\right)}=\prod_{\ell=1}^{n} \frac{\bar{q}\left(y_{\ell-1}, y_{\ell}\right)}{q\left(y_{\ell-1}, y_{\ell}\right)}
$$

Proof Observe first that since $x, y \in C$, there exists a feasible path $y=z_{0}, z_{1}, \ldots, z_{k-1}, z_{k}=x$ from $y$ to $x$. Furthermore, Condition (b) also implies

$$
\prod_{\ell=1}^{k} \frac{\bar{q}\left(z_{\ell-1}, z_{\ell}\right)}{q\left(z_{\ell-1}, z_{\ell}\right)} \prod_{\ell=1}^{m} \frac{\bar{q}\left(x_{\ell-1}, x_{\ell}\right)}{q\left(x_{\ell-1}, x_{\ell}\right)}=1=\prod_{\ell=1}^{k} \frac{\bar{q}\left(z_{\ell-1}, z_{\ell}\right)}{q\left(z_{\ell-1}, z_{\ell}\right)} \prod_{\ell=1}^{n} \frac{\bar{q}\left(y_{\ell-1}, y_{\ell}\right)}{q\left(y_{\ell-1}, y_{\ell}\right)}
$$


since $z_{0}, z_{1}, \ldots, z_{k-1}, z_{k}=x_{0}, x_{1}, \ldots, x_{m}$ and $z_{0}, z_{1}, \ldots, z_{k-1}, z_{k}=y_{0}, y_{1}, \ldots, y_{n}$ are both feasible paths starting and ending at state $y$. After dividing the far-left and far-right terms of (1) by their common multiple, we find

$$
\prod_{\ell=1}^{m} \frac{\bar{q}\left(x_{\ell-1}, x_{\ell}\right)}{q\left(x_{\ell-1}, x_{\ell}\right)}=\prod_{\ell=1}^{n} \frac{\bar{q}\left(y_{\ell-1}, y_{\ell}\right)}{q\left(y_{\ell-1}, y_{\ell}\right)}
$$

which proves our claim. $\diamond$

Conditions (a) and (b) from Theorem 1.1 were conjectured on pg. 64 of [4] to be sufficient for two Markov chains to be similar over $C$. Theorem 1.1 also generalizes Theorem 2 of [3] where two sufficient conditions equivalent to (a) and (b) are given for two birth-death processes to be similar. In fact (as also pointed out in [4]) the authors of [3] further assume an associated Stieltjes moment problem has a unique solution, but the arguments we provide here show this extra assumption is not needed to establish similarity, once Conditions (a) and (b) are known to hold.

Interestingly, in [3] the authors also show (see Theorem 1 of [3]) that similarity of two birth-death processes over a communicating class $C$ implies Conditions (a) and (b) of Theorem 1.1 must hold on $C$, i.e. that these are also necessary conditions for similarity on $C$. The author has tried showing that - as also conjectured in [4] Conditions (a) and (b) are also necessary for similarity to hold over $C$ in this more general context, but currently it is not clear this is the case.

\section{Preliminaries}

Our approach involves working with the Laplace transforms of the transition functions associated with both $X$ and $\bar{X}$, as opposed to working with the transition functions directly. For each $x, y \in E$, we define for $\operatorname{Re}(\alpha)>0$

$$
\pi_{x, y}(\alpha):=\int_{0}^{\infty} e^{-\alpha t} p_{x, y}(t) d t, \quad \bar{\pi}_{x, y}(\alpha):=\int_{0}^{\infty} e^{-\alpha t} \bar{p}_{x, y}(t) d t
$$


The first proposition in this section yields a useful expression for each Laplace transform $\pi_{x, y}$, $x, y \in E$.

Proposition 2.1 For each $x, y \in E$,

$$
\pi_{x, y}(\alpha)=\frac{\mathbb{E}_{x}\left[\int_{0}^{\tau_{x}} e^{-\alpha t} \mathbf{1}(X(t)=y) d t\right]}{1-\mathbb{E}\left[e^{-\alpha \tau_{x}}\right]}, \quad \operatorname{Re}(\alpha)>0
$$

where $\tau_{x}:=\inf \{t \geq 0: X(t-) \neq X(t)=x\}$, with $X(t-):=\lim _{s \uparrow t} X(s)$ for each $t>0$.

Proof Fix any two states $x, y \in E$, where possibly $x=y$. Then for each $t \geq 0$,

$$
\begin{aligned}
p_{x, y}(t) & =\mathbb{P}_{x}\left(X(t)=y, \tau_{x}>t\right)+\mathbb{P}_{x}\left(X(t)=y, \tau_{x} \leq t\right) \\
& =\mathbb{P}\left(X(t)=y, \tau_{x}>t\right)+\int_{0}^{t} p_{x, y}(t-s) d \mathbb{P}_{x}\left(\tau_{x} \leq s\right) .
\end{aligned}
$$

Integrating both sides of (3) by $e^{-\alpha t} d t, \operatorname{Re}(\alpha)>0$, over $[0, \infty)$ and simplifying gives $(2)$. $\diamond$

Equation (2) simplifies even further when $x=y$, as the next corollary illustrates.

Corollary 2.1 For each state $x \in E$,

$$
\pi_{x, x}(\alpha)=\frac{1}{(q(x)+\alpha)\left(1-\mathbb{E}_{x}\left[e^{-\alpha \tau_{x}}\right]\right)}, \quad \operatorname{Re}(\alpha)>0 .
$$

Proof Starting with the numerator of (2), we find

$$
\mathbb{E}_{x}\left[\int_{0}^{\tau_{x}} e^{-\alpha t} \mathbf{1}(X(t)=x) d t\right]=\mathbb{E}_{x}\left[\int_{0}^{T_{1}} e^{-\alpha t} d t\right]=\frac{1}{\alpha} \mathbb{E}_{x}\left[1-e^{-\alpha T_{1}}\right]=\frac{1}{q(x)+\alpha}
$$

which proves our claim, once we plug $(4)$ into $(2) . \diamond$

The final result of this section shows that the Laplace transforms $\pi_{x, y}, x, y \in E$, can be expressed in terms of expected values of random products governed by a CTMC $\{\tilde{X}(t) ; t \geq 0\}$ on $E$ having a 
rate matrix $\tilde{\mathbf{Q}}$ that satisfies the following properties: (i) for any two states $x, y \in E, x \neq y, \tilde{q}(x, y)>0$ if and only if $q(y, x)>0$, and (ii) for each $x \in E$, we have $-\tilde{q}(x, x)=\sum_{x \neq y} \tilde{q}(x, y)=-q(x, x)$.

In order to make use of $\tilde{X}$ and $\tilde{\mathbf{Q}}$, we must assume $X$ and $\bar{X}$ do not have any absorbing states, and there may be many situations (such as in [3]) where this is an unreasonable assumption. Fortunately, we can remove absorbing states in a harmless manner by adding new states in the following way: if a state $z_{0} \in E$ is an absorbing state, simply create another new state $z_{0}^{\prime}$, and extend $\mathbf{Q}$ by choosing positive values for $q\left(z_{0}, z_{0}^{\prime}\right)$ and $q\left(z_{0}^{\prime}, z_{0}\right)$, and setting $q\left(z_{0}\right):=-q\left(z_{0}, z_{0}^{\prime}\right), q\left(z_{0}^{\prime}\right):=-q\left(z_{0}^{\prime}, z_{0}\right)$.

Theorem 2.1 Suppose $x, y \in E$, where $x \neq y$. Then the Laplace transform $\pi_{x, y}$ of $p_{x, y}$ satisfies

$$
\pi_{x, y}(\alpha)=\pi_{x, x}(\alpha) \mathbb{E}_{y}\left[e^{-\alpha \tilde{\tau}_{x}} \prod_{\ell=1}^{\tilde{\eta}_{x}} \frac{q\left(\tilde{X}_{\ell}, \tilde{X}_{\ell-1}\right)}{\tilde{q}\left(\tilde{X}_{\ell-1}, \tilde{X}_{\ell}\right)}\right], \quad \operatorname{Re}(\alpha)>0
$$

where $\tilde{\tau}_{x}:=\inf \{t>0: \tilde{X}(t-) \neq \tilde{X}(t)=x\}$, and $\tilde{\eta}_{x}:=\inf \left\{n \geq 1: \tilde{X}_{n}=x\right\}$.

This result is a generalization of Theorem 1.2 from [2], since (i) in [2] we only work with chains that do not explode with probability one, and (ii) in [2] Equation (5) is only shown to hold when $\alpha>0$, but here we find it is still valid when we only assume $\operatorname{Re}(\alpha)>0$.

Proof Fix two states $x, y \in E, x \neq y$, and note that for $\operatorname{Re}(\alpha)>0$,

$$
\begin{aligned}
\pi_{x, y}(\alpha) & =\frac{1}{(q(x)+\alpha)\left(1-\mathbb{E}_{x}\left[e^{-\alpha \tau_{x}}\right]\right)}(q(x)+\alpha) \mathbb{E}_{x}\left[\int_{0}^{\tau_{x}} e^{-\alpha t} \mathbf{1}(X(t)=y) d t\right] \\
& =\pi_{x, x}(\alpha)(q(x)+\alpha) \mathbb{E}_{x}\left[\int_{0}^{\tau_{x}} e^{-\alpha t} \mathbf{1}(X(t)=y) d t\right]
\end{aligned}
$$

so to complete the proof, it remains to show that

$$
(q(x)+\alpha) \mathbb{E}_{x}\left[\int_{0}^{\tau_{x}} e^{-\alpha t} \mathbf{1}(X(t)=y) d t\right]=\mathbb{E}_{y}\left[e^{-\alpha \tilde{\tau}_{x}} \prod_{\ell=1}^{\tilde{\eta}_{x}} \frac{q\left(\tilde{X}_{\ell}, \tilde{X}_{\ell-1}\right)}{\tilde{q}\left(\tilde{X}_{\ell-1}, \tilde{X}_{\ell}\right)}\right]
$$

The argument used to establish (6) is just slightly different from that used to prove Theorem 1.2 of [2], in that we do not make use of a clearing model. Starting with the right-hand-side of (6), we 
observe after summing over all finite feasible paths from $y$ to $x$ under $\tilde{\mathbf{Q}}$ that

$$
\begin{aligned}
& \mathbb{E}_{y}\left[e^{-\alpha \tilde{\tau}_{x}} \prod_{\ell=1}^{\tilde{\eta}_{x}} \frac{q\left(\tilde{X}_{\ell}, \tilde{X}_{\ell-1}\right)}{\tilde{q}\left(\tilde{X}_{\ell-1}, \tilde{X}_{\ell}\right)}\right] \\
= & \sum_{n=1}^{\infty} \sum_{x_{0}=y, x_{n}=x, x_{1}, \ldots, x_{n-1} \neq x}\left[\prod_{\ell=1}^{n} \frac{q\left(x_{\ell}, x_{\ell-1}\right)}{\tilde{q}\left(x_{\ell-1}, x_{\ell}\right)}\right]\left[\prod_{\ell=1}^{n} \frac{\tilde{q}\left(x_{\ell-1}\right)}{\tilde{q}\left(x_{\ell-1}\right)+\alpha}\right] \prod_{\ell=1}^{n} \frac{\tilde{q}\left(x_{\ell-1}, x_{\ell}\right)}{\tilde{q}\left(x_{\ell-1}\right)} \\
= & \frac{q(x)+\alpha}{q(y)+\alpha} \sum_{n=1}^{\infty} \sum_{x_{0}=y, x_{n}=x, x_{1}, \ldots, x_{n-1} \neq x}^{n} \prod_{\ell=1}^{n} \frac{q\left(x_{\ell}, x_{\ell-1}\right)}{q\left(x_{\ell}\right)+\alpha} \\
= & \frac{q(x)+\alpha}{q(y)+\alpha} \sum_{n=1}^{\infty} \mathbb{E}_{x}\left[e^{-\alpha T_{n}} \mathbf{1}\left(X_{n}=y, \eta_{x}>n\right)\right] \\
= & (q(x)+\alpha) \sum_{n=1}^{\infty} \mathbb{E}_{x}\left[e^{-\alpha T_{n}} \mathbf{1}\left(X_{n}=y, \eta_{x}>n\right) \frac{1}{\alpha} \frac{\alpha}{q(y)+\alpha}\right] \\
= & (q(x)+\alpha) \sum_{n=1}^{\infty} \mathbb{E}_{x}\left[e^{-\alpha T_{n}} \mathbf{1}\left(X_{n}=y, \eta_{x}>n\right) \frac{1}{\alpha} \mathbb{E}\left[\left[1-e^{-\alpha\left(T_{n+1}-T_{n}\right)} \mid X_{0}, \ldots, X_{n}, T_{1}, \ldots, T_{n}\right]\right]\right. \\
= & (q(x)+\alpha) \mathbb{E}_{x}\left[\mathbb{E}_{x^{\prime}}\left[\sum_{n=1}^{\infty} \mathbf{1}\left(\eta_{x}>n\right) \int_{T_{n}}^{T_{n+1}} e^{-\alpha t} \mathbf{1}(X(t)=y) d t \mid X_{0}, \ldots, X_{n}, T_{1}, \ldots, T_{n}\right]\right] \\
= & (q(x)+\alpha) \mathbb{E}_{x}\left[\int_{0}^{\tau_{x}} e^{-\alpha t} \mathbf{1}(X(t)=y) d t\right]
\end{aligned}
$$

which establishes (6). Here the fifth equality follows from observing

$$
\mathbb{E}\left[1-e^{-\alpha\left(T_{n+1}-T_{n}\right)} \mid X_{0}, \ldots, X_{n}, T_{1}, \ldots, T_{n}\right] \mathbf{1}\left(X_{n}=y\right)=\frac{\alpha}{\alpha+q(y)} \mathbf{1}\left(X_{n}=y\right)
$$

with probability one, and the sixth equality is a consequence of both Fubini's theorem, as well as $e^{-\alpha T_{n}} \mathbf{1}\left(X_{n}=y, \eta_{x}>n\right)$ being a measurable function of $T_{0}, \ldots, T_{n}, X_{1}, \ldots, X_{n}$. $\diamond$

Remark In the proof of Theorem 2.1, we are implicitly making use of the fact that at each return to a state $x \in E$, the total number of transitions made by $X$ before this return time must be finite: if the chain explodes before we revisit $x$, then $\tau_{x}=\infty$, meaning $x$ is not revisited. 


\section{Proof of Theorem 1.1}

We first show, for each $x \in C$, that $\bar{\pi}_{x, x}(\alpha)=\pi_{x, x}(\alpha)$ on $\operatorname{Re}(\alpha)>0$. This of course proves $\bar{p}_{x, x}(t)=p_{x, x}(t)$ for $t \geq 0$, since two continuous functions defined on $[0, \infty)$ that share the same Laplace transform must be equal. Starting with Corollary 2.1, we find

$$
\pi_{x, x}(\alpha)=\frac{1}{(q(x)+\alpha)\left(1-\mathbb{E}_{x}\left[e^{-\alpha \tau_{x}}\right]\right)}=\frac{1}{(\bar{q}(x)+\alpha)\left(1-\mathbb{E}_{x}\left[e^{-\alpha \bar{\tau}_{x}}\right]\right)}=\bar{\pi}_{x, x}(\alpha)
$$

where $\bar{\tau}_{x}:=\inf \{t>0: \bar{X}(t-) \neq \bar{X}(t)=x\}$, since from Conditions (a) and (b) of Theorem 1.1, we get

$$
\begin{aligned}
\mathbb{E}_{x}\left[e^{-\alpha \tau_{x}}\right] & =\sum_{n=2}^{\infty} \sum_{\substack{x_{0}, \ldots, x_{n}: \\
x_{0}=x, x_{n}=x, x_{1}, \ldots, x_{n-1} \neq x}} \prod_{\ell=1}^{n} \frac{q\left(x_{\ell-1}, x_{\ell}\right)}{q\left(x_{\ell-1}\right)+\alpha} \\
& =\sum_{n=2}^{\infty} \sum_{\substack{x_{0}, \ldots, x_{n}: \\
x_{0}=x, x_{n}=x, x_{1}, \ldots, x_{n-1} \neq x}} \prod_{\ell=1}^{n} \frac{\bar{q}\left(x_{\ell-1}, x_{\ell}\right)}{\bar{q}\left(x_{\ell-1}\right)+\alpha}=\mathbb{E}_{x}\left[e^{-\alpha \bar{\tau}_{x}}\right] .
\end{aligned}
$$

It remains to show $\bar{\pi}_{x, y}(\alpha)=c_{x, y} \pi_{x, y}(\alpha)$, for $x, y \in C, x \neq y$, and $\operatorname{Re}(\alpha)>0$, where

$$
c_{x, y}=\prod_{\ell=1}^{n} \frac{\bar{q}\left(x_{\ell}, x_{\ell-1}\right)}{q\left(x_{\ell}, x_{\ell-1}\right)}
$$

is the product formed by any chosen feasible path $x=x_{n}, x_{n-1}, \ldots, x_{1}, x_{0}=y$ from $x$ to $y$ under Q. Starting with (5), we find after applying both (7) and Lemma 1.1 that

$$
\begin{aligned}
\bar{\pi}_{x, y}(\alpha) & =\bar{\pi}_{x, x}(\alpha) \mathbb{E}_{y}\left[e^{-\alpha \tilde{\tau}_{x}} \prod_{\ell=1}^{\tilde{\eta}_{x}} \frac{\bar{q}\left(\tilde{X}_{\ell}, \tilde{X}_{\ell-1}\right)}{\tilde{q}\left(\tilde{X}_{\ell-1}, \tilde{X}_{\ell}\right)}\right] \\
& =\pi_{x, x}(\alpha) \mathbb{E}_{y}\left[e^{-\alpha \tilde{\tau}_{x}}\left(\prod_{\ell=1}^{\tilde{\eta}_{x}} \frac{\bar{q}\left(\tilde{X}_{\ell}, \tilde{X}_{\ell-1}\right)}{q\left(\tilde{X}_{\ell}, \tilde{X}_{\ell-1}\right)}\right)\left(\prod_{\ell=1}^{\tilde{\eta}_{x}} \frac{q\left(\tilde{X}_{\ell}, \tilde{X}_{\ell-1}\right)}{\tilde{q}\left(\tilde{X}_{\ell-1}, \tilde{X}_{\ell}\right)}\right)\right] \\
& =c_{x, y} \pi_{x, x}(\alpha) \mathbb{E}_{y}\left[e^{-\alpha \tilde{\tau}_{x}} \prod_{\ell=1}^{\tilde{\eta}_{x}} \frac{q\left(\tilde{X}_{\ell}, \tilde{X}_{\ell-1}\right)}{\tilde{q}\left(\tilde{X}_{\ell-1}, \tilde{X}_{\ell}\right)}\right] \\
& =c_{x, y} \pi_{x, y}(\alpha)
\end{aligned}
$$


which proves Theorem 1.1.

Acknowledgments The author gratefully acknowledges the support of the National Science Foundation, via grant NSF-CMMI-1435261. Furthermore, the author thanks an anonymous referee for providing comments that helped simplify the proofs of Proposition 2.1 and Corollary 2.1, as well as improve the overall presentation of the material in this note.

\section{References}

[1] Anderson, W.J. (1991). Continuous-time Markov chains: an applications-oriented approach. Springer, New York.

[2] Buckingham, P. and Fralix, B. (2015). Some new insights into Kolmogorov's criterion, with applications to hysteretic queues. Markov Processes and Related Fields 21, 339-368.

[3] Lenin, R.B., Parthasarathy, P.R., Scheinhardt, W.R.W., and van Doorn, E.A. (2000). Families of birth-death processes with similar time-dependent behaviour. Journal of Applied Probability 37, $835-849$.

[4] Pollett, P.K. (2001). Similar Markov chains. Journal of Applied Probability 38A, 53-65. 